\title{
Wege zur guten Kommunikation
}

\section{Anforderungen an System- und Kommunikationstechnologien}

Wie man zu einer technischen Kommunikationslösung gelangt, bei der das Verhalten von separaten Systemen gekoppelt ist, beschreibt dieser Beitrag. Die dabei notwendigerweise zu berücksichtigen Rahmenbedingungen und die damit verbundenen Entscheidungskriterien werden anhand des Beispiels „Integration von Messdaten ins ERP-System“ näher betrachtet. Weiterhin werden die Anforderungen an Kommunikationstechnologien formuliert, um derartige Lösungsszenarien geeignet zu unterstützen.

SCHLAGWÖRTER Kommunikation / Integration / Leitfaden / MES

\section{Ways to Good Communication}

How to get a technical communication application where the behavior of separate systems are connected is described in this contribution. The constraints and the decision criteria which have to be considered are illustrated by means of an example ,integration of measured data into ERP system“. Furthermore, the requirements to communication technologies are defined that are necessary to support these application scenarios.

KEYWORDS Communication / Integration / Guideline / MES 
ANSGAR MÜNNEMANN, BASF SE

n der Automatisiserungstechnik wird bei Diskussionen zum Themenkomplex Kommunikation oftmals sehr stark auf die Technik zur Datenübertragung fokussiert. Es werden die Vorzüge von $4 . .20 \mathrm{~mA}$, Feldbus, Ethernet, OPC, Wireless, etc. diskutiert und die Probleme beziehungsweise Herausforderungen beschrieben, die sich bei dem jeweiligen Technologieeinsatz ergeben.

Als Anwender und Betreiber von Automatisierungssystemen und deren Verbindung in umfangreichen Netzwerken muss man sich dem Thema Kommunikation stärker von der Nutzenseite nähern. Kommunikation hat in dieser Sichtweise immer ein bestimmtes Ziel, nämlich Produktions- und Geschäftsprozesse und Arbeitsabläufe zu optimieren. Dieses Ziel kann in Abhängigkeit der jeweils vorhandenen bzw. verfügbaren Systeme, Technologien und organisatorischen Prozesse auf unterschiedliche Weise erreicht werden.

Die Aufgabe besteht also darin, einen geeigneten Weg zu finden, der die gewünschte Kommunikation im Sinne der Zielerreichung ermöglicht. Dies lässt sich plakativ auch mit einem aus dem Schulunterricht bekannten Beispiel verdeutlichen: den kommunizierenden Röhren (siehe Bild 1). Das Verhalten des synchronen Ansteigen des Wasserpegels wird im Fall der „natürlich“ kommunizierenden Röhren durch die physikalische Verbindung der Gefäße erreicht. Ist eine solche Verbindung nicht vorhanden, muss dieses Verhalten mit Hilfe einer technischen Kommunikation durch den Austausch geeigneter Informationen (Füllstandmessung) und zusätzlicher Steuerungseinrichtungen realisiert werden.

Da eine Kommunikation auf verschiedenen Wegen erreicht werden kann, ist es notwendig, die Möglichkeiten bewerten zu können, um auf diese Weise eine „gute“ Kommunikation zu erreichen. Aus Betreibersicht führt dies letztendlich immer zur Betrachtung des Aufwands, der mit der Einführung und dem Erhalt einer Kommunikation im Lebenszyklus der beteiligten Systeme verbunden ist. Wobei insbesondere die Heterogenität der Systeme und die sich ändernden Rahmenbedingungen $\mathrm{zu}$ bedenken sind.
Im Folgenden wird das Vorgehen, wie man von einer Kommunikationszielstellung zu einer Technologieauswahl gelangen kann, näher betrachtet. Die dabei offensichtlich werdende Notwendigkeit, die Kommunikation ganzheitlich zu betrachten, ermöglicht es dann, generelle Anforderungen an System- und Kommunikationstechnologien zu formulieren, die auf dem Weg zur guten Kommunikation benötigt werden.

\section{LEITFADEN FÜR KOMMUNIKATIONSANWENDUNGEN}

Als Beispiel, anhand dessen man den Weg von einer Kommunikationszielstellung $\mathrm{zu}$ einer technischen Kommunikationslösung gut darstellen kann, wird im folgenden das Szeanrio Datenübertragung vom Messgerät ins ERP-System (Enterprise Resource Planning) herangezogen.

\subsection{Das Zielverhalten}

Das eine klare und präzise Zielvorstellung den wesentlichen Ausgangspunkt für den Weg zu einer Kommunikationslösung darstellt, zeigt sich, wenn man das Thema Übernahme von Messwertinformationen ins ERP-System mit unterschiedlichen Personenkreisen diskutiert. Die in Bild 2 dargestellten Lösungsszenarien verdeutlichen dies beispielhaft.

Eine Konkretisierung der Zielstellung hinsichtlich

- Mengengerüst der benötigten Daten (z.B.: 30 Messwerte)

- Art der Daten bzw. Verwendung der Daten (z.B. Verbrauchsbuchungen im ERP)

- Übertragungszeit und Häufigkeit (z.B. täglich)

- Qualität der Information (Genauigkeit, Verlässlichkeit, Verfügbarkeit)

würde bereits dazu führen, die Lösungsszenarien „Bürokommunikation“ und „Direkte Kopplung“ aus Bild 2 anders zu bewerten. 


\subsection{Systemstruktur}

Nach Klärung der konkreten Zielstellung ist es erforderlich, sich mit den vorhandenen bzw. möglichen Systemstrukturen zu beschäftigen, die an möglichen Lösungsszenarien beteiligt sind. Dazu müssen sowohl die jeweiligen Funktionen der Systeme als auch die Informationsflüsse zwischen den Systemen verstanden werden. In dem Beispiel ERP-Datenkopplung ist es u.a. wichtig zu wissen, ob die Messwerte bereits in ein PLS eingebunden sind, ob das PLS über Schnittstellen verfügt, die den Zugriff auf diese Daten erlauben, und ob es im Unternehmen eventuell bereits Systeme gibt, die Messwerte in ERP-Verbrauchsbuchungen umwandeln.

Die folgenden drei Punkte sollten bei der Betrachtung des Themas Systemstruktur bei jedem Kommunikationskonzept berücksichtigt werden:

1 | Verwendung von vorhandener Infrastruktur

2 | Nutzung zentraler Systeme und Funktionen

3 | Schnittstellen und Kommunikationsverbindungen zu Systemen

Eine typische Systemlandschaft der Prozessindustrie zeigt Bild 3. Die im PLS anfallenden Prozessdaten werden über Schnittstellensysteme in ein PIMS (Plant Information Management System) übertragen und dort für MES-Anwendungen (Manufacturing Execution System) [1,2] wie beispielsweise die Ermittlung von Einsatzstoffverbrauchsmengen und deren Übertragung ins ERP zur Verfügung gestellt. Die Automatisierungsnetzwerke sind zur Officeund ERP-Welt durch Firewall-Technologie abgesichert.

\subsection{Verantwortlichkeiten}

Im dritten Schritt sollten die Verantwortlichkeiten für die Systeme innerhalb der Unternehmensorganisation betrachtet werden. In unserem Beispiel wäre evtl. die zentrale IT für die Office-Welt und das ERP-System verantwortlich, die SupplyChain bestimmt die Abläufe und Prozesse im ERP, das zentrale Engineering betreibt die PIMS und MES Systeme und die Prozessleitsysteme liegen in der Verantwortung der jeweiligen Betriebsbetreuung. Verbunden mit den Verantwortlichkeiten ist nicht nur die Frage, wo man den jeweiligen Ansprechpartner im Unternehmen für Kommunikationsfragen zu dem jeweiligen System findet, sondern auch unterschiedliche Philosophien hinsichtlich

- Nutzung der Systeme und Kommunikation mit diesen (z.B. der Zugriff auf Daten nur durch authentifizierte Personen)

- Änderungsmanagement (ob dies jeder Zeit, einmal im Jahr oder nur bei ausreichend großem Bedarf erfolgt; werden die Änderungen zentral und für alle Systeme gleichzeitig oder dezentral mit Berücksichtigung individueller Anforderungen durchgeführt)

- Wartungs- und Störungskonzepte (wie erfolgt die Reaktion, Priorisierung und Eskalation bei Störfällen)
- LifeCycle-Strategien (in der IT werden Systemwechsel typischerweise alle 3-5 Jahre, in der Automatisierungstechnik ca. alle 15 Jahre durchgeführt)

Das sind wichtige Aspekte, die auf dem Weg zu einer Kommunikation berücksichtigt werden müssen.

\subsection{Information}

Im 4. Schritt auf dem Weg zu einer Kommunikation müssen die Daten bzw. Informationen, die kommuniziert werden sollen, näher betrachtet werden. Dabei gilt es, verschiedene Aspekte einer Information zu berücksichtigen. Zum einen die Identität bzw. Adressierung, also wie die Information in dem jeweiligen System angesprochen wird. Wichtig ist ebenfalls der Typ der Daten, also ob es sich um einen einzelnen Datenwert eines bestimmten Typs handelt oder um Zeitreihen, Ereignisse oder komplexe Datenstrukturen. Neben diesen elementaren Aspekten von Daten ist für eine korrekte Interpretation auch die Meta-Information notwendig, also z.B. im Fall eines Messwertes die Einheit, der Messbereich, der Status und der Zeitstempel. Die Meta-Information alleine reicht für eine Interpretation jedoch noch nicht aus, sondern man benötigt außerdem den Kontext der Information, also z.B. um welche PLT-Stelle es sich bei dem Messwert handelt, wo die Messung in der Anlage platziert ist oder welche Stoffströme während der Messung in der Anlage vorlagen.

Betrachten wir die exemplarische Systemlandschaft aus Bild 3 dahingehend, wo diese Informationsaspekte $\mathrm{zu}$ ein und der selben Information überall zu finden sind, so zeichnet sich ein sehr heterogenes Bild: In jedem System können Adressierung und Datentyp und insbesondere Meta- und Kontextinformation unterschiedlich definiert sein oder festgelegt werden. Es ist eine wesentliche Voraussetzung für eine funktionierende Kommunikation, dass neben der eigentlichen Datenwertübertragung auch diese Informationsaspekte in sich konsistent verwaltet werden.

\subsection{Kommunikationstechnologie}

Die Betrachtung der einzusetzenden Kommunikationstechnologien sollte erst dann erfolgen, wenn die anderen vorhergehnd ausgeführten Aspekte von Kommunikation geklärt sind, da anderfalls wesentliche Rahmenbedinungen, die den Einsatz spezifischer Technologien mitbestimmen, übersehen werden können. Bei der Betrachtung der vorhandenen bzw. einzusetzenden Kommunikationstechnologien empfiehlt es sich, entlang der verschiedenen Ebenen einer Kommunikationstechnologie (vgl. ISO-OSI-Schichtenmodell [3]) vorzugehen. In Bild 4 ist eine vereinfachte Unterteilung in physikalische Schicht (Kabel, Stecker, Ex-Schutz, etc.), Transportschicht (Adressierung, Weiterleitung von Daten- 


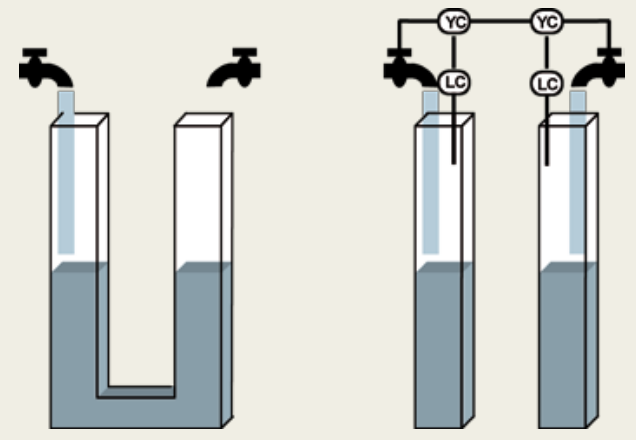

BILD 1: (links) „Natürlich“ kommunizierende Röhren und (rechts) .technisch“ kommunizierende Röhren
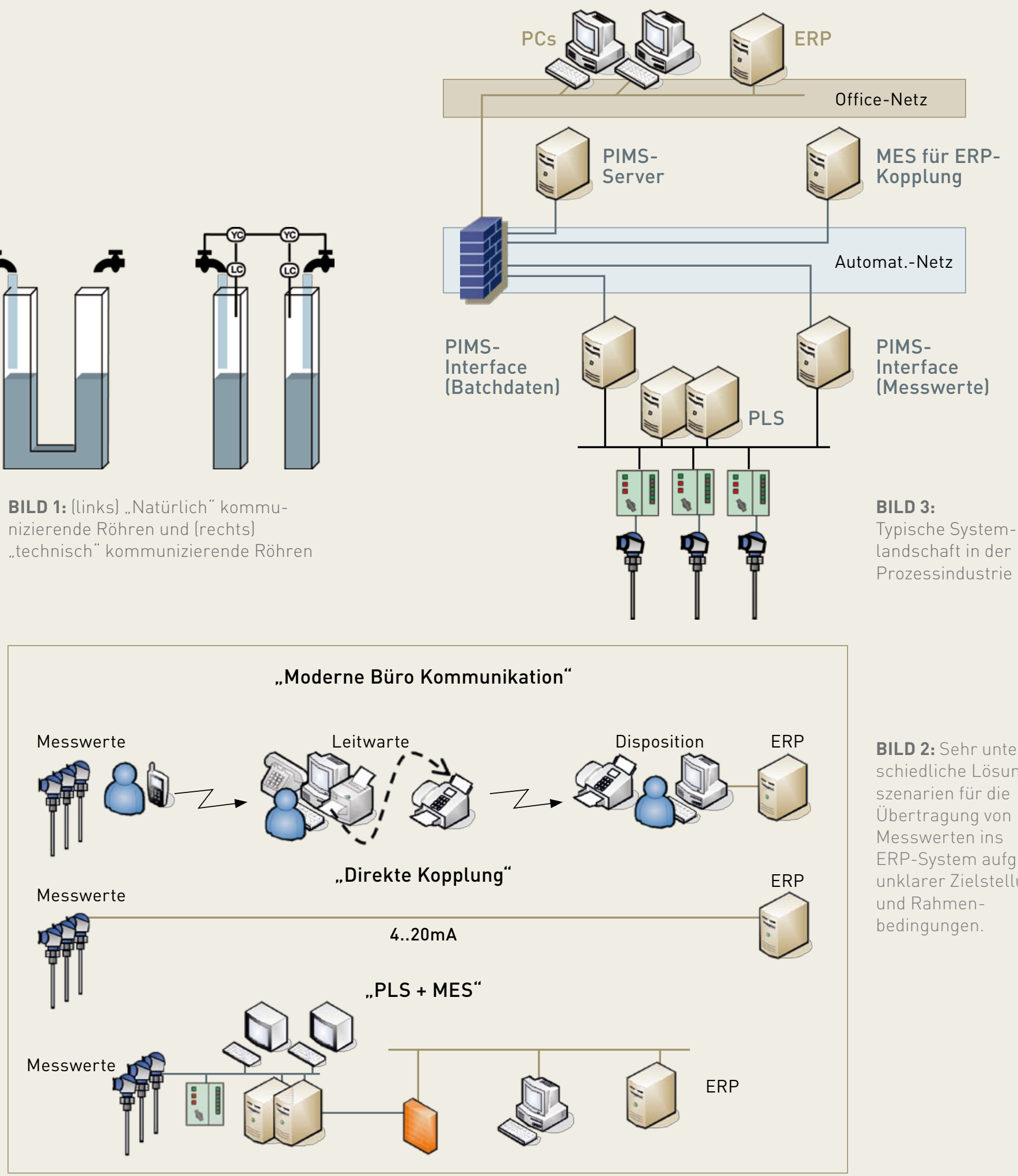

BILD 2: Sehr unterschiedliche Lösungsszenarien für die Übertragung von Messwerten ins ERP-System aufgrund unklarer Zielstellung und Rahmenbedingungen. 
strömen) und Anwendungsschicht (Datenstrukturen, Dienste, usw.) dargestellt.

Für eine genauere Betrachtung der jeweiligen Schicht kann man noch die drei Implementierungsaspekte: Protokoll, Schnittstelle und Konfiguration näher hinterfragen. Die Protokollimplementierung definiert die jeweiligen Stati der Kommunikationspartner, die auszutauschenden Nachrichtenköpfe und Datenstrukturen. Die Schnittstellenimplementierung beschreibt, über welche Funktionen in einem System die entsprechenden Kommunikationsdienste aufgerufen werden können. Und der Implementierungsaspekt Konfiguration definiert das Wie und Wo von möglichen Konfigurationseinstellungen der Kommunikation. Die genauere Betrachtung dieser Aspekte kann sehr hilfreich sein, um z.B. den Umfang eines Standards genauer zu hinterfragen. So werden beispielsweise in den Feldbus-Standards [4] die Protokolle festgelegt, die Schnittstellen der Feldbus-IOKarten können aber sehr unterschiedlich sein [5].

Für das Beispiel der ERP-Datenintegration könnten sich so u.a. die folgenden Fragen ergeben, die näher betrachtet werden müssen:

- Wie sieht in der Anwendungsschicht die Schnittstellensyntax der ERP-Kommunikation aus?

- Inwieweit ist die Transportschicht der PIMS internen Kommunikation firewall-tauglich?

- Wie erfolgt die Konfiguration der TCP/IP-Ports der PIMS internen Kommunikation?

- Sind bereits Netzanschlüsse der physikalischen Schicht des Automatisierungsnetzwerkes vorhanden?

\section{SYSTEM- UND KOMMUNIKATIONSTECHNOLOGIEN}

Nachdem im vorherigen Abschnitt ein Leitfaden in fünf Schritten von der Kommunikationszielstellung bis zur Kommunikationstechnologie aufgestellt wurde, bleibt weiterhin die Frage bestehen, ob es sich bei einer gemäß des Leitfadens erarbeiteten Kommunikationslösung dann auch um eine gute Kommunikation handelt. Wie in der Einleitung erwähnt, ist „gut“ aus Betreibersicht immer eine Frage des Aufwands für den Kommunikationslebenszyklus. Je geringer dieser Aufwand ist (bei gleicher Funktionalität), umso besser ist die Kommunikation.

\subsection{Stand der Technik}

Um den Stand der Technik anschaulich zu beschreiben, wird wieder das Beispiel ERP-Datenintegration als Referenz herangezogen und bezüglich heute üblicher Umsetzungstechnologien näher erläutert.

Die Feld-Kommunikation von Messgerät und PLS soll an dieser Stelle nicht näher betrachtet werden. Das PLS stellt somit die Datenquelle der Messwertinformationen dar. Um die Kommunikation zwischen PLS und PIMS zu ermöglichen, wird auf einem eigenen PLS-Koppelsystem ein OPC-Server installiert (siehe Bild 5). Für diesen muss konfiguriert werden, welche Messstellen aus dem PLS darüber adressierbar sein sollen. Die übertragenen OPC-Datenpunkte bestehen aus der OPC-Adresse und dem Wert. Weitere Informationen wie Einheit o.ä. sind nicht direkter Bestandteil.

Bevorzugt auf derselben Hardware (um eine OPCNetzkommunikation zu vermeiden) wird eine OPC-DAKlientenkopplung [6] zum PIMS installiert. Im PIMS muss festgeleget werden, welche OPC-Datenpunkte als was für PIMS-Datenpunkte (Name, Datentyp, Einheit, ...) übertragen werden sollen. Hier haben wir das erste typisches Beispiel für den Verlust von Meta-Information: die Einheit des Messpunktes ist im PLS noch bekannt, muss im PIMS aber nachkonfiguriert werden, da die Übertragung nicht Bestandteil der üblichen Kommunikationstechnik ist.

Die proprietäre Kommunikation zwischen PIMS-Interface und PIMS-Server fällt immer dann auf, wenn sie mit anderen Netzwerkkomponenten des Betreibers (hier im Beispiel die Firewall) kompatibel sein muss. Dann kann man feststellen, dass die Kompatibilität manchmal nur teilweise gegeben ist und sich vor allem produkt- und versionsbedingt ändern kann. Konkret heißt dies, dass die Einstellungen in der Firewall bei einem Upgrade der PIMS-Software evtl. angepasst werden müssen, weil der Hersteller seine proprietäre TCP/ IP-Kommunikation geändert hat.

Der Zugriff auf die Daten im PIMS erfolgt über ODBCDatenbankkommunikation. Da es sich bei ODBC um eine Vereinheitlichung der Anwendungsschnittstelle, nicht aber der Übertragung handelt, muss auf dem MES der (versionsabhängige) aktuelle ODBC-Treiber für das PIMS bereitgestellt werden. Für die eigentliche Datenabfrage muss auch die Tabellenstruktur der Daten im PIMS-System bekannt und im MES korrekt eingestellt werden. Bezüglich Meta-Informationen wie z.B. dem Status des Datenpunktes (z.B.: gültig, ungültig, etc.) muss auch die PIMS-spezifische Semantik für eine korrekte Interpretation im MES festgelegt werden.

In dem MES-System müssen nun aus den Messstellenwerten durch Zuordnung zum jeweiligen Produktionsauftrag und entsprechender Berechnungsvorschrift (z.B. Integration der Durchflussmessung über Dauer der Auftragsbearbeitung oder Umrechnungen von Einheiten etc.) die Verbrauchs- und Produktionsrückmeldungen für das ERPSystem generiert werden. Dazu muss das MES Kontextinformationen über den jeweiligen Anlagenstatus haben (welches Produkt wird gerade auf welchen Anlagenteilen hergestellt), Informationen, die oftmals im PLS nur implizit vorhanden sind, aber nicht ohne weiteres einem Kommunikationspartner zur Verfügung stehen.

Die Kommunikation in Richtung ERP, auch wenn sie hier beispielhaft auf offenen Web-Service-,,Standards“ wie HTTP/SOAP basiert, muss in spezifischer ERPSystemausprägung im MES konfiguriert werden. Selbst wenn ERP und MES darauf aufbauende Standards wie B2MML [7] unterstützen würden, ist aufgrund der Unverbindlichkeit dieser Standards hinsichtlich Semantik 


\begin{tabular}{|c|c|c|c|c|}
\hline \multirow[b]{2}{*}{$\begin{array}{l}\text { Ebenen der } \\
\text { Kommunikation }\end{array}$} & \multirow{2}{*}{$\begin{array}{r}\text { Implementierungs- } \\
\text { aspekte }\end{array}$} & Protokoll & Schnittstelle & Konfiguration \\
\hline & & $\begin{array}{l}\text { Codierung } \\
\text { Ablauf } \\
\text { Zustände }\end{array}$ & $\begin{array}{c}\text { Funktionen (Dienste) } \\
\text { Parameter } \\
\text { Datenstrukturen }\end{array}$ & $\begin{array}{l}\text { Parameter } \\
\text { Dateien } \\
\text { Werkzeuge }\end{array}$ \\
\hline $\begin{array}{l}\text { Anwendungs- } \\
\text { schicht }\end{array}$ & $\begin{array}{l}\text { Datenformate } \\
\text { Dienste } \\
\text { Syntax, Semantik }\end{array}$ & & $\begin{array}{l}\text { Schnittstellen- } \\
\text { syntax für ERP- } \\
\text { Kommunikation }\end{array}$ & \\
\hline $\begin{array}{l}\text { Transport- } \\
\text { schicht }\end{array}$ & $\begin{array}{l}\text { Adressierung } \\
\text { Routing, Fehler- } \\
\text { behandlung }\end{array}$ & $\begin{array}{l}\text { PIMS interne } \\
\text { Kommunikation } \\
\text { Firewall tauglich? }\end{array}$ & & $\begin{array}{l}\text { Wie erfolgt die } \\
\text { Einstellung von } \\
\text { festen TCP/IP-Ports? }\end{array}$ \\
\hline $\begin{array}{l}\text { Physikalische } \\
\text { Schicht }\end{array}$ & $\begin{array}{l}\text { Kabel, Stecker } \\
\text { Ex-Schutz } \\
\text { Wireless }\end{array}$ & Anschluss für Al & latisierungsnetzwerk in & Betrieb vorhanden? \\
\hline
\end{tabular}

BILD 4: Analysematrix für Kommunikationstechnologien

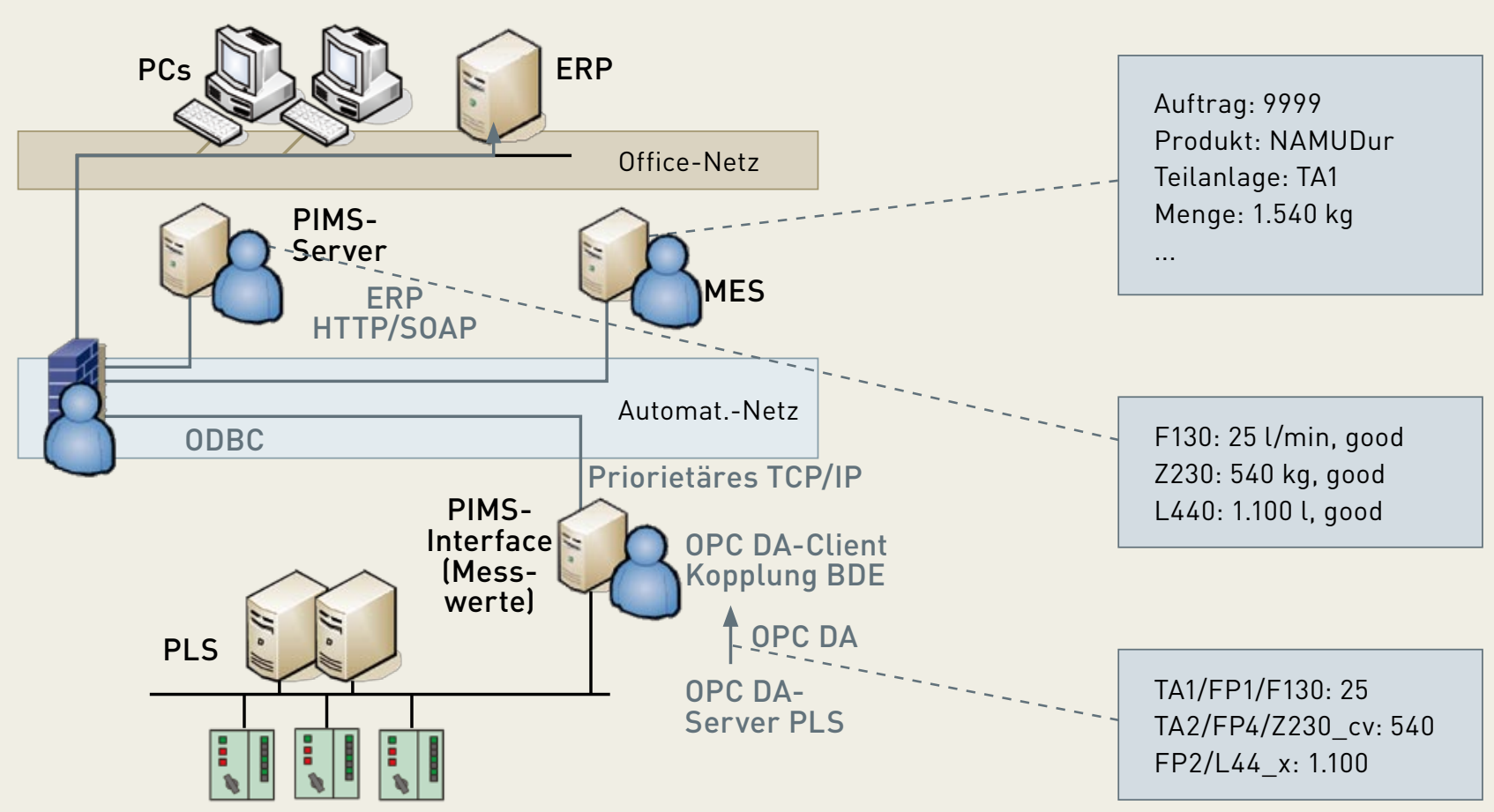

BILD 5: Stand der Technik der Kommunikationswege für eine Datenübertragung vom Messgerät zum ERP-System (das Mensch-Symbol steht für wiederkehrende manuelle Tätigkeiten im Lebenszyklus der Kommunikationslösung). 
und Dienstemechanismen eine individuelle Konfigurationsanpassung unvermeidlich.

Die verschiedenen Technologiewechsel, die nicht durchgängige Übertragung von Metainformationen und die fehlende Offenheit der Systeme sowie implizit vorhandene Kontextinformationen verdeutlichen, dass derartige Kommunikationslösungen einen nicht unerheblichen Aufwand in ihrem Lebenszyklus generieren.

\subsection{Das Ziel}

Das Ziel liegt auf der Hand: Der Aufwand im Lebenszyklus von Kommunikationsanwendungen soll weiter reduziert werden, damit die Kommunikation „,besser“ wird. Dabei muss man insbesondere in der Prozessindustrie mit ihren typisch langen Anlagenlaufzeiten grundsätzlich immer von einer heterogenen Systemlandschaft ausgehen, so dass hier ,einfache“ SingleVendor-Lösungen keinen wirklichen Lösungsansatz darstellen.

Ein Gutes-Kommunikation-Szenario könnte etwa wie folgt aussehen: Dem PLS teilt der Anwender durch Konfiguration mit, dass sämtliche Durchflussmessungen in ein zentrales PIMS übertragen werden sollen. Das PLS erfragt bei den anderen Teilnehmern im Automatisierungsnetz freie PIMS-Kapazitäten. Entsprechend den Rückmeldungen wird die Kommunikation selbstständig aufgebaut und dabei die vollständige Information inklusive Meta- und Kontextinformation übertragen. Beispielsweise wäre der Kontext der Stoff, dessen Durchfluss von dem Sensor erfasst wird, und der Anlagenbezug des Durchflussmessers. Darüber hinaus gehende Detailkonfigurationen sollten nicht erforderlich sein.

Für die Erstellung von ERP-Rückmeldungen auf Basis der Messwertinformationen sollte wiederum nur eine sehr begrenzte funktionale Konfiguration erforderlich sein, die keine Kenntnis der Details der Kommunikationstechniken voraussetzt. Jeder weitere Informationsaustausch erfolgt automatisch, weil Meta- und Kontextinformationen einheitlich ausgetauscht und interpretiert werden können. So kann z.B. die Teilanlagenkennung aus dem ERP vom MES verwendet werden, um in dem PIMS über den Stoffkontext die zugehörigen Durchflussmessungen zu identifizieren und daraus die Einsatzstoffverbrauchsmengen für die ERP-Rückmeldung abzuleiten.

\subsection{Anforderungen}

Aus dem beschriebenen Stand der Technik, der Soll-Vorstellung eines optimierten Lebenszyklus von Kommunikationsanwendungen und aus den einzelnen Aspekten des Leitfades für Kommunikation lassen sich Anforderungen an System- und Kommunikationstechnologien im Umfeld der Automatisierungstechnik ableiten:

\section{Offener Informationszugang}

Ganz oben auf der 5-Punkteliste steht der offene Informationszugang in den verschiedenen Automatisierungssystemen auch für Meta- und Kontextinformationen. Dabei muss man über die initiale Aufgabenstellung eines Automatisierungssystems hinausdenken und den vollständigen Zugang zu Informationen sicherstellen, da bei der Einführung eines solchen Systems nur teilweise bekannt sein kann, was für zukünftige Automatisierungslösungen zusätzlich noch kommuniziert werden muss.

\section{Kommunikationsintelligenz}

Damit Kommunikation zwischen den Systemen möglichst selbstständig und gleichzeitig flexibel ablaufen kann, wird mehr Kommunikationsintelligenz zwischen den Systemen benötigt. Das lässt sich durch eine Diensteorientierung nicht nur der Kommunikation, sondern auch der Systemfunktionalitäten erreichen [8].

\section{Eindeutige Semantik}

Verbunden mit der Kommunikationsintelligenz ist die Festlegung von eindeutiger Semantik in der Kommunikation zwischen Automatisierungssystemen- und Komponenten auch unterschiedlicher Hersteller.

\section{Durchgängigkeit}

Im Rahmen einer evtl. zukünftigen Ethernet-FeldbusTechnologie wird oft die Durchgängigkeit der physikalischen Netzwerktechnologie als Vorteil genannt. Sicherlich ein wichtiger Punkt. Aus Anwendungssicht ist jedoch die Durchgängigkeit der Anwendungsschicht auch auf Basis unterschiedlicher Transport- oder physikalischer Schichten viel entscheidender.

\section{Informationsorientierung}

Als fünften Punkt, der die Voraussetzung für eine gute Kommunikation schafft, kann eine generelle Stärkung der Informationsorientierung bei der Umsetzung von Automatisierungslösungen genannt werden, da hierbei auch bei den heutigen Technologien zu wenig darauf geachtet wird. Oftmals liegt der Fokus auf den in einem System zu realisierenden Funktionen, und die Auswirkung auf die weiterzuverarbeitende Information wird vernachlässigt.

\section{FAZIT}

Kommunikation ist ein wesentlicher Bestandteil von praktisch jeder Automatisierungslösung. Die Konzeption und Umsetzung einer Kommunikationslösung sollte sich immer an einer ganzheitlichen Sichtweise auf Kommunikation orientieren. Dazu wurde in dem Beitrag ein Leitfaden entwickelt, der in fünf Schritten bei der Auffindung eines geeigneten Kommunikationsweges unterstützen kann.

Dieser Leitfaden weist auf wesentliche Aspekte von Kommunikation hin, die in den heute verfügbaren Technologien zur Umsetzung von Kommunikationsanwendungen nur unzureichend berücksichtigt werden, was letzt- 
endlich zu unnötig hohen Aufwändungen für die Einrichtung und den Betrieb derartiger Anwendungen führt.

Um dem Ziel einer guten Kommunikation näher zu kommen, müssen Hersteller und Anwender von Automatisierungssystemen gemeinsam daran arbeiten, die in dem Beitrag formulierten Anforderungen an System- und Kommunikationstechnologien weiter auszuarbeiten und sowohl in den Standardisierungsprozess als auch in die jeweilige Systementwicklung einfließen zu lassen.

MANUSKRIPTEINGANG 06.12.2009

\section{AUTOR}

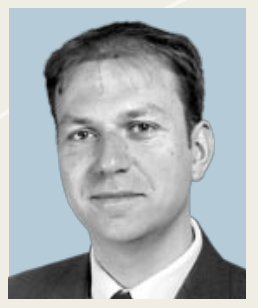

Dr.-Ing. ANSGAR MÜNNEMANN (geb. 1971), Studium der Physik, RWTH Aachen, Promotion am Lehrstuhl für Prozessleittechnik, seit 2005 Mitarbeiter der BASF SE, Leiter der Fachgruppe MES im Bereich Engineering und Maintenance. Arbeitsschwerpunkte sind die Konzeption und Begleitung von Automatisierungslösungen aus dem Bereich Systemintegration, Datenerfassung, -analyse und -bewertung, Plant AssetManagement und KPI-Monitoring.

BASF SE,

Carl-Bosch-Straße 38,

D-67056 Ludwigshafen,

E-Mail:ansgar.muennemannabasf.com

\section{REFERENZEN}

[1] NAMUR: NA 94: MES - Funktionen und Lösungsbeispiele der Betriebsleitebene

[2] IEC 62264: Enterprise-control system integration

[3] DIN ISO 7498: Information technology - Open Systems Interconnection - Basic Reference Model

[4] IEC 61158: Digital data communications for measurement and control - Fieldbus for use in industrial control systems

[5] T. Kraußer, U. Epple, C. Haus: UniFeBu - Ein Universeller Feldbuszugang, AUTOMATION 2008

[6] OPC Foundation: OPC Data Access, 2003

[7] WBF: B2MML Business to Manufacturing Markup Language

[8] OASIS Reference Model for Service Oriented Architecture. www.oasis-open.org.

\section{NAMUR-Signal reicht}

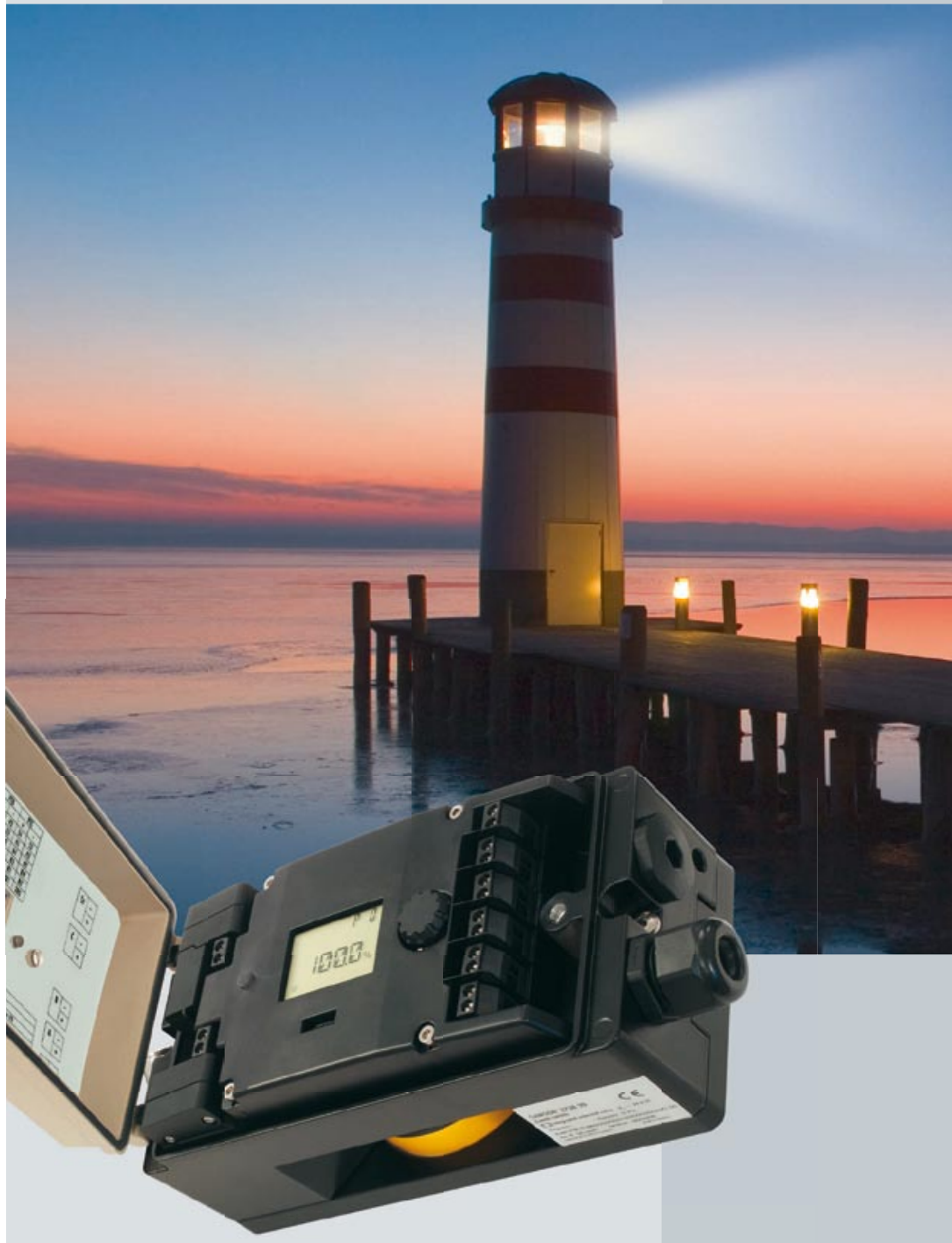

Der elektronische Grenzsignalgeber Typ 3738 mit Magnetventil bietet die ideale Lösung für Schwenkarmaturen. Als erstes Gerät seiner Art ermöglicht es die Speisung von Elektronik und berührungslosem Wegsensor aus dem NAMUR-Signal. So kann die Verkabelung unverändert bleiben. Trotzdem bietet es eine Fülle zusätzlicher Funktionen bei der Automatisierung von Auf/Zu-Armaturen, wie zum Beispiel die Konfigurierung per Tastendruck, Selbstabgleich und Diagnose. Justierarbeiten entfallen ganz. Dank integrierter Lufftührung braucht das Gerät keine externe Verrohrung. Einfach anschrauben, Knopf für Selbstabgleich drücken, fertig.

Der neue Grenzsignalgeber macht die Auf/Zu-Armatur smart und kompakt. 